\title{
Bodily Maps of Emotion in Major Depressive Disorder
}

\author{
Naomi Lyons ${ }^{1}$ - Alexa Strasser ${ }^{1} \cdot$ Bernard Beitz $^{2} \cdot$ Tobias Teismann $^{3} \cdot$ Thomas Ostermann $^{1} \cdot$ Laura Anderle $^{4}$. \\ Johannes Michalak ${ }^{1}$
}

Accepted: 8 December 2020 / Published online: 28 December 2020

(c) The Author(s) 2020

\begin{abstract}
Background Emotions play a central role in mental disorder and especially in depression. They are sensed in the body, and it has recently been shown in healthy participants that these sensations can be differentiated between emotions. The aim of the current study was to assess bodily sensations for basic emotions induced by emotion eliciting pictures in depression. Methods 30 healthy controls (HC), 30 individuals suffering from Major depressive disorder (MDD) without medication use (MDDnm) and 30 individuals with MDD with medication use (MDDm) were shown emotional and neutral pictures and were asked to paint areas in an empty body silhouette where they felt an increase or decrease in activation. Body sensation maps were then calculated and statistical pattern recognition applied.

Results Results indicated statistically separable activation patterns for all three groups. MDDnm showed less overall activation than $\mathrm{HCs}$, especially in sadness and fear, while MDDm showed stronger deactivation for all emotions than the other two groups.

Conclusions We could show that emotion experience was associated with bodily sensations that are weaker in depression than in healthy controls and that antidepressant medication was correlated with an increased feeling of bodily deactivation. Results give insights into the relevance for clinicians to acknowledge bodily sensations in the treatment of depression.
\end{abstract}

Keywords Bodily sensation maps $\cdot$ BSM $\cdot$ Depression $\cdot$ Medication $\cdot$ IAPS $\cdot$ Emotion induction $\cdot$ Replication

The perception and regulation of emotion has specific importance for both mental wellbeing and psychological disorders (Berking and Wupperman 2012). Even though definitions of emotion vary widely, it is generally agreed upon the notion that all emotions encompass behavioral, experiential and visceral changes (Gendron and Barrett 2009). The relevance of visceral changes is often discussed not only as an epiphenomenon (i.e., I am scared, therefore my heart is

Supplementary Information The online version of this article (https://doi.org/10.1007/s10608-020-10195-0) contains supplementary material, which is available to authorized users.

Naomi Lyons

naomi.lyons@uni-wh.de

1 Witten/Herdecke University, Alfred-Herrhausen-Straße 44, 58448 Witten, Germany

2 University of Wuppertal, Wuppertal, Germany

3 Ruhr-University Bochum, Bochum, Germany

4 Westphalian University of Applied Sciences, Gelsenkirchen, Germany racing) but also as a prerequisite of emotions (i.e., My heart is racing, therefore I am scared) (e.g., Niedenthal 2007). One of the first to claim that emotions are a result of altered body states was William James (1884). His idea of distinct physiological activation patterns for different emotions was criticized at that time, as no physiological system was known to be complex enough to account for distinct emotions (e.g., Cannon 1927). However, the idea of an interplay between body and mind for the formation of emotions remained a central concept (e.g., Craig 2003; Damasio and Carvalho 2013). Today, it is widely accepted that at the bodily and neurological levels (e.g., Kreibig 2010; Saarimäki et al. 2018), a complex system exists that can process even subtle bodily changes quickly and accurately enough to allow a distinct activation pattern for emotional experience.

Research on the connection between emotion experience and physiological activation is often limited to objective physiological markers that are only modestly correlated with the actual experience of emotions (e.g., Mauss et al. 2005). Still, as Mauss and Robinson (2009) suggest, it is important to consider all levels of emotion experience. As such, the 
examination of the subjective experience of bodily sensations in relationship to emotion was presented by Nummenmaa et al. (2014) with the emBODY tool. Participants (overall $N=701$ in 5 experiments) were shown two silhouettes of bodies during an emotion induction, and were asked to color the body regions for which they perceived an increase or decrease in activity while viewing each stimulus. The authors were able to show distinct patterns of activation for basic and complex emotions over five experiments. In one experiment, participants from Finland, Sweden and Taiwan received emotion words and were asked to paint body sensation maps (BSM) in accordance with these emotions. In further experiments, varying emotion induction techniques were applied. In the final experiment, the task was reversed and participants were asked to indicate emotional states that were presented in BSMs. Nummenmaa and colleagues were able to show that the same emotion-specific patterns of activation and deactivation emerged across all experiments. Torregrossa and colleagues (2018) studied BSMs in patients diagnosed with schizophrenia and showed that they differed from those of healthy adults; in patients, activation patterns were indistinguishable between emotions.

Whether individuals with depression show a different pattern than healthy adults has not yet been investigated. However, several findings from both emotion and interoception studies suggest that altered emotion processing and perception of bodily sensations in depression occurs. A meta-analysis of emotion processing in depression (Bylsma et al. 2008) showed that depressed individuals have a reduced reactivity to negative and positive stimuli in emotion-eliciting experimental designs. The findings are not unequivocal (e.g., Sloan and Sandt 2010), yet, low positive affectivity remains a stable finding in emotion-eliciting design studies in depression.

Furthermore, support for an altered perception of bodily signals comes from interoception studies. Interoception is defined as "the sense of the physiological condition of the entire body" (Craig 2002, p. 655) and is often measured via the interoceptive accuracy (IA), i.e., how accurate someone is able to - for example - count one's heartbeat (e.g., Schandry 1981). Several authors have investigated IA in depression with inconsistent findings that in sum point towards a low IA in moderately depressed individuals and a better IA in severely depressed individuals (for review, see Eggart et al. 2019). Different confounding variables are discussed that might cause this effect. Among those are the increased comorbidity with anxiety in addition to a heightened preoccupation with bodily signals in anxiety (Paulus and Stein 2010). Individuals with anxiety disorders are assumed to be increasingly aware of - and monitor - those subtle bodily changes that are associated with an emotion, appraise them as threatening and in its most extreme form they might lead to a panic attack (e.g., Farb et al. 2015; Ginzburg et al. 2013). Studies that controlled for anxiety have found reduced
IA in depressed individuals (e.g., Furman et al. 2013) and overall, results suggest that in depression, IA is reduced and associated with less positive affectivity (Eggart et al. 2019).

In their meta-analysis, Eggart and colleagues additionally found a possibly confounding effect of antidepressant medication on IA in depression. This finding is in line with a study assessing effects of antidepressant medication on emotional and interpersonal experiences. In a sample of $N=1829$ New Zealanders, feelings of emotional numbness and reduction of positive feelings were reported among other negative side effects due to the medication (Read 2014).

On the basis of these findings, the purpose of the current study was twofold. First, we aimed to conceptually replicate Nummenmaa and colleagues' (2014) results with a different emotion induction method. As such, the current study used emotion-eliciting pictures taken from the International Affective Picture System (IAPS et al. 2008) to induce basic emotions of anger, disgust, happiness, sadness, and fear, as well as a neutral emotional state. Our second aim was to examine bodily sensations associated with emotions in depression. The findings of Torregrossa and colleagues (2018) led to the hypothesis that in depression, BSMs would be indistinguishable and show less activation overall. In addition, due to a possibly confounding effect of antidepressant medication on IA and findings on reduced emotional feelings in patients taking antidepressant medication, we explored the impact of antidepressant medication on bodily sensations associated with emotions. The third, exploratory aim was therefore to assess whether bodily sensations differ between depressed individuals with or without antidepressant medication use.

\section{Methods}

\section{Participants}

Following requirement for authors 2 ("Authors must collect at least 20 observations per cell") from Simmons and colleagues (2011, p. 1363), participants included in the study were $n=30$ with current major depressive disorder without antidepressant intake (MDDnm), $n=30$ with current major depressive disorder with antidepressant use (MDDm), and $n=30$ never depressed, healthy controls (HC). The MDDnm group was recruited via newspaper, Facebook groups and through word-of-mouth. The MDDm group was recruited from two German psychiatric outpatient wards (in Witten/ Herdecke and Hemer). All groups were diagnosed via the German version of the Structured Clinical Interview for DSM-IV, Axis I Disorders (SCID-I; Wittchen et al. 1997) by masters-level students who were extensively trained and supervised by the second author. Inclusion criteria for the MDD groups were current major depressive disorder, 
normal or corrected to normal vision and the ability to read and write in the German language. Exclusion criteria were current suicidality, psychotic symptoms, somatoform disorder, chronic pain syndrome or chronic physical diseases. Pain and somatoform disorders were excluded because they might have an impact on the bodily felt activation and deactivation. Three participants with anomalies in BSMs (drawings of question marks or no coloring at all) were excluded from the analysis.

The HC group was recruited from Witten/Herdecke University through word-of-mouth. Participants could receive course credit upon request. HCs were excluded from the study if they had a history of depression. Otherwise, the same inclusion criteria as for the MDD groups and exclusion of pain symptoms applied to HCs.

The study was approved by the local ethics committee of the University (application \#29/2016) and was conducted in accordance with the Declaration of Helsinki. All participants signed an informed consent form after being instructed about their risks and rights.

\section{Materials and Procedure}

\section{Mood Induction}

Emotion stimuli were taken from the IAPS (Lang et al. 2008). In order to induce discrete emotions via the pictorial stimuli, pictures were chosen according to empirically derived classification of emotional content of IAPS pictures from Mikels and colleagues (2005): Chosen pictures were selected to induce anger (picture numbers: 2100 , $6360,9180,9560)$, fear $(1052,1301,1930,6370)$, happiness $(2304,2310,2360,2550)$, sadness $(2141,2205,2800$, 2900 ) and disgust (7361, 7380, 9300, 9390). In recognition of the higher vulnerability of the depressed sample, pictures with moderate arousal ratings were used (3.63-6.44; total range: 1 to 9) according to the values reported by Lang and colleagues (2008). Additionally, neutral pictures also taken from IAPS stimuli $(7001,7004,7009,7010)$ were presented, resulting in $6 \times 4$ pictures in total. The pictures had a maximum size of $1024 \times 768$ pixels (approx. $7 \times 5 \mathrm{~cm}$ ) and were presented at a size of 3.74 inches $(9.5 \mathrm{~cm})$ in pseudorandomized order.

\section{EmBODY}

On a 13.3-inch display (approx. $34 \mathrm{~cm}$ ), two human silhouettes were presented on the right and left side, while instructions were given in the middle of the screen. In each trial, a picture taken from IAPS was presented underneath the instructions, which prompted participants to indicate where in the body they sensed more or less activation when they saw the picture. The setup of the emBODY tool was taken from the Nummenmaa Lab website (http://emotion.utu.fi/). Participants colored the two silhouettes with the mouse according to their activation and deactivation. For activation, i.e., when the participant felt an increase or stronger activity due to the emotion picture, they were asked to color the left silhouette in red. For deactivation, i.e., when they felt a decrease or weakening of activity, the right silhouette was to be colored blue. Painting was dynamic, so that repeated coloring of one region intensified the color. The diameter of the painting tool was 12 pixels and each body was represented by 50,364 pixels in a $171 \times 522$ pixels rectangle. On the 13.3-inch display, it resulted in a height of approx. $11 \mathrm{~cm}$ and width of approx. $4 \mathrm{~cm}$ with a brush diameter of approx. $3 \mathrm{~mm}$. Participants were free to take as much time as they needed. Paint intensity for the finished images ranged from 0 to 100 and was stored in matrices. Participants were allowed to leave silhouettes empty if no activation or deactivation was present.

\section{Beck Depression Inventory II (BDI II; Beck et al. 1996)}

Group differences in intensity of depressive symptoms were assessed as a control measure with the BDI II. The BDI is a self-report questionnaire of 21 items measuring depression severity. The items are presented as groups of statements, answered on a 4-point Likert scale ranging from 0 to 3. The total sum score ranges from 0 to 63 with higher scores reflecting a higher degree of depression. The German version has good internal consistency for depressed $(\alpha=0.84)$ and community samples $(\alpha=0.89)$ (Kühner et al. 2007).

\section{Data Analysis}

Possible confounding parameters that might account for diverging results between the depressed and healthy samples were analyzed. Among those, age and body mass index were tested between the three groups with a univariate ANOVA. Gender, current deployment, smoking and exercising were analyzed between the three categorical groups with $\chi^{2}$ Test and years of education with the Kruskal-Wallis $H$ Test for non-metric measure.

BSM data analysis was conducted in accordance with the recommendations of the Nummenmaa Lab. Analysis script can be viewed on https://github.com/bernb/embody_depre ssion/. First, deactivation and activation were combined and data from the four IAPS-picture stimuli for each emotion averaged subjectwise, so that one body map resulted for each emotion in each participant. Brush size was smoothed with a Gaussian kernel with a 15-pixel filter and coloring outside the body area was masked. In order to compare the activation from resulting body maps pixelwise to zero, mass-independent $t$-tests were calculated in random effects analyses. False discovery rate analysis (FDR) was applied at an alpha 
level of 0.05 to account for an accumulated alpha error in mass comparisons. $T$-values in the resulting $t$-maps indicated significant pixel intensity and therefore statistically significant felt bodily changes due to the emotion induction. In order to analyze differences between the groups, difference maps were created by subtracting $t$-values of the respective body maps. FDR was then applied.

To replicate Nummenmaa and colleagues' (2014) results on significantly distinct activation patterns for different emotions and to test whether individuals with depression show different activation patterns, statistical pattern recognition with linear discriminant analysis (LDA) was performed for each group individually. At least $n=20$ units per predictor are recommended (Hahs-Vaughn 2017, p. 286f). In the current study $n=120$ units per predictor (four pictures per emotion per participant) were taken. Dimensionality was first reduced to 30 components with principal component analysis and then LDA was applied. The accuracy of the model was determined with fivefold cross-validation where classifiers were trained to recognize all emotional states against all (complete classification). Cross-validation was conducted 100 times to obtain a mean accuracy score with standard deviation and to statistically test accuracy against chance level. LDA classification for HCs was applied to MDDm and MDDnm in order to examine whether emotion classification is identical for $\mathrm{HCs}$ and depressed individuals.

\section{Results}

Descriptive statistics are presented in Table 1.

Groups significantly differed in their average depressive symptomatology $(F(2,81)=66.99, p<0.001)$. Post-hoc tests with Bonferroni correction indicated that $\mathrm{HC}$ differed significantly from both depressive groups with an average BDI score of $3.43(S D=2.87)$; $\operatorname{MDDm}(M=31.29, S D=11.90)$ and MDDnm $(M=28.38, S D=9.62)$ did not differ significantly from each other.

\section{BSMs in Healthy Controls}

Bodily activation for $\mathrm{HC}$ for the six emotions is presented in Fig. 1a. Color bars indicate $t$-values that are significantly different from zero after controlling for alpha error accumulation with FDR. Red and yellow colors represent activation while blue colors represent deactivation. $T$-values ranged from $t=-2.71$ to 10.68 . The percentage of deactivation in total painted area was $24 \%$ and no longer visible after applying the FDR. For number of painted pixels overall see Table 2. Results indicate that BSMs can be classified according to the corresponding emotion with an accuracy rate of $36 \%$. Compared to a classification accuracy of $17 \%$ in the case of chance, results
Table 1 Demographic data of the three groups: Major depressive disorder - medicated (MDDm), Major depressive disorder - non-medicated (MDDnm) and control group (HC)

\begin{tabular}{|c|c|c|c|c|c|}
\hline & $\begin{array}{l}\text { MDDm } \\
N=30 \\
M / \%(S D)\end{array}$ & $\begin{array}{l}\text { MDDnm } \\
N=30 \\
M / \%(S D)\end{array}$ & $\begin{array}{l}\mathrm{HC} \\
N=30 \\
M / \%(S D)\end{array}$ & Statistical test $\mathrm{t}^{\mathrm{a}}$ & $p$ \\
\hline Age (years) & $43.6(11.7)$ & $45.0(14.2)$ & $44.4(15.7)$ & $F(2,89)=0.08$ & .92 \\
\hline Gender ( $\%$ female) & 53.3 & 56.7 & 60.0 & $\chi^{2}(2, N=90)=0.271$ & .87 \\
\hline BMI & $26.3(6.8)$ & $26.8(7.7)$ & $24.9(4.3)$ & $F(2,85)=0.66$ & .52 \\
\hline Years of education & $10.8(1.6)$ & $11.4(2.0)$ & $11.6(1.5)$ & $H(2)=4.77$ & .09 \\
\hline Current deployment & & & & $\chi^{2}(2, N=90)=17.86$ & .21 \\
\hline - Full time (\%) & 33.3 & 16.7 & 43.3 & & \\
\hline - Part time (\%) & 13.3 & 20.0 & 26.7 & & \\
\hline - Unemployed (\%) & 20.0 & 16.7 & 3.3 & & \\
\hline - Other $(\%)$ & 33.4 & 46.6 & 26.7 & & \\
\hline Smoker (\% yes) & 40.0 & 16.7 & 16.7 & $\chi^{2}(2, N=90)=5.97$ & .05 \\
\hline Sports (\% yes) & 40.0 & 46.7 & 56.7 & $\chi^{2}(2, N=90)=2.27$ & .32 \\
\hline \multicolumn{6}{|l|}{ Antidepressants } \\
\hline - Venlaflaxin (\%) & 22.0 & & & & \\
\hline - Escitalopram (\%) & 6.5 & & & & \\
\hline - Mirtazapin (\%) & 10.0 & & & & \\
\hline - Citalopram (\%) & 6.5 & & & & \\
\hline - Others $(\%)$ & 16.0 & & & & \\
\hline - N/A (\%) & 39.0 & & & & \\
\hline
\end{tabular}

$B M I$ body mass index, N/A not available

${ }^{a}$ ANOVA for age and BMI; Kruskal-Wallis $H$ Test for years of education; $\chi^{2}$ Test for gender, current deployment, smoker/non-smoker and sports/non-sports 
(a)

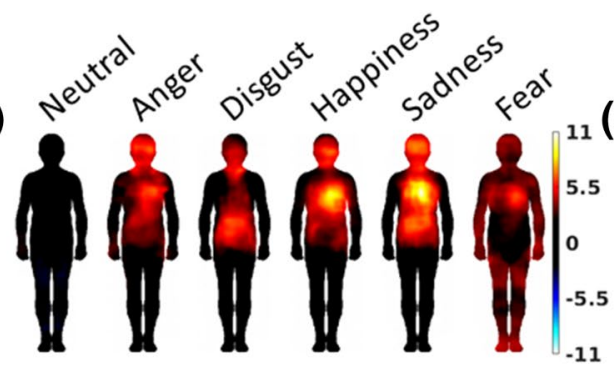

(d)

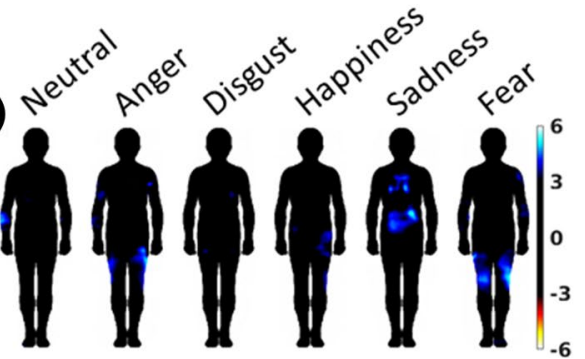

(b)
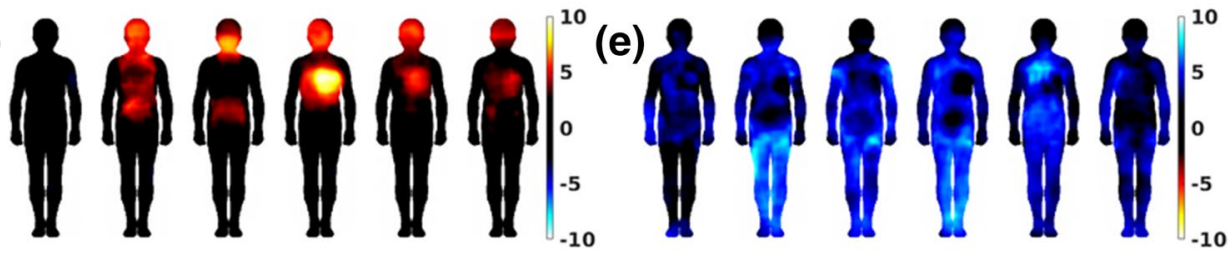

(c)
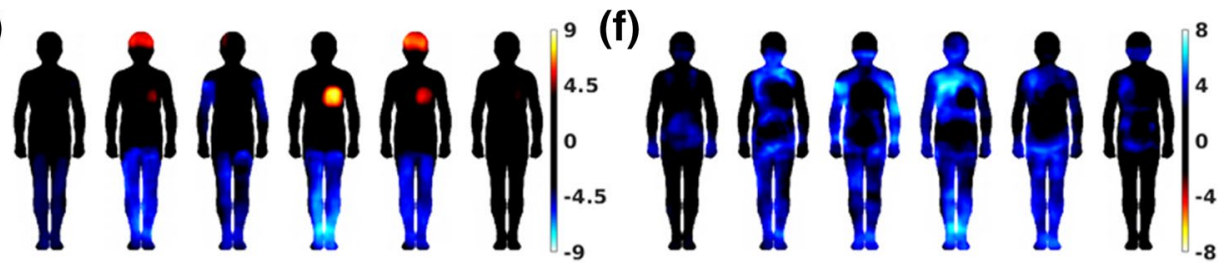

Fig. 1 Body sensation maps for a healthy controls (HC) with FDR threshold of 2.20 , b patients with major depressive disorder - nonmedicated (MDDnm) with FDR threshold of 2.56, $\mathrm{c}$ patients with MDD - medicated (MDDm) with FDR threshold of 3.28. Difference- maps d HC-MDDnm with FDR threshold of 2.67, e HC-MDDm with FDR threshold of 1.83 and $\mathbf{f}$ MDDnm-MDDm with FDR threshold of 2.03

Table 2 Number of pixels colored for healthy controls (HC), individuals with Major depressive disorder- non-medicated (MDDnm) and Major depressive disorder- medicated (MDDm) for each emotion after FDR and proportion to each other in \%

\begin{tabular}{lllllcc}
\hline & HC & MDDnm & MDDm & $\begin{array}{l}\text { Activation of MDDnm } \\
\text { relative to HC in \% }\end{array}$ & $\begin{array}{l}\text { Activation of MDDm } \\
\text { relative to HC in \% }\end{array}$ & $\begin{array}{l}\text { Activation of MDDm } \\
\text { relative to MDDnm in \% }\end{array}$ \\
\hline Neutral & 7,803 & 22,257 & 3,240 & $41.52 \%$ & 285.24 & 686.94 \\
Anger & 35,708 & 28,316 & 23,867 & $66.84 \%$ & 79.30 & 118.64 \\
Disgust & 23,580 & 32,212 & 14,873 & $63.07 \%$ & 136.61 & 216.58 \\
Happiness & 34,161 & 24,946 & 21,328 & $62.43 \%$ & 73.03 & 116.96 \\
Sadness & 28,704 & 27,501 & 17,719 & $61.73 \%$ & 95.81 & 155.21 \\
Fear & 61,144 & 888 & 14,384 & $23.52 \%$ & 1.45 & 6.17 \\
\hline
\end{tabular}

indicate good classification accuracy that is comparable to that found by Nummenmaa and colleagues (2014). The confusion matrix indicates that a neutral emotional state was classified most accurately, followed by happiness and disgust (for all emotions, see Fig. 2a, Table S1). Anger pictures were correctly classified in only $9 \%$ of the cases, which is below chance level. Therefore, an additional analysis was performed with anger excluded. The accuracy rate now reached $44 \%$ with a chance rate of $20 \%$ (Table S4 and Figure S6).

\section{BSMs for Individuals Suffering from Major Depression}

BSMs for MDDnm and MDDm are presented in Fig. 1b, c. Color bars ranged from $t=-.23-9.75$ in MDDnm and $t=-.97-8.03$ in MDDm. The percentage of deactivation in the total painted area was $38.76 \%$ for MDDnm and $63.34 \%$ for MDDm (for the number of painted pixels and proportion to each other, see Table 2). Classification accuracy for depressed samples was similar to that of HC (MDDnm: 


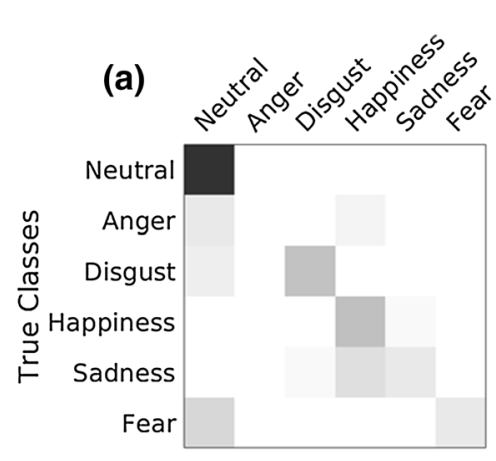

(b)

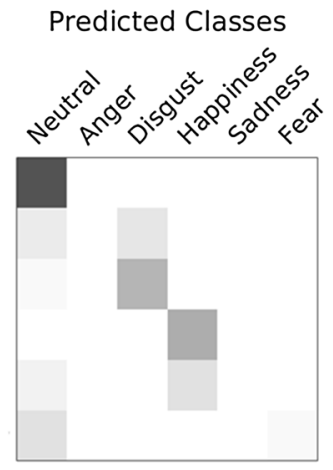

(c)

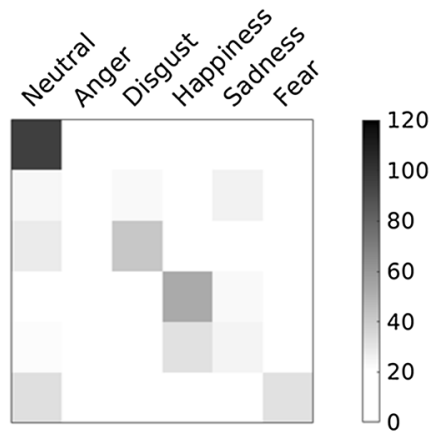

Fig. 2 Confusion matrices for a healthy controls (HC), b major depressive disorder - non-medicated (MDDnm), $\mathbf{c}$ MDD - medicated (MDDm) between predicted and true classifications

$M=34 \%$ with anger and $43 \%$ without anger; MDDm: $M=36 \%$ with anger and $45 \%$ without anger; for additional information see Tables S5, S6, Figures S7, S8). Therefore, BSMs are readily distinguishable by individuals suffering from depression. Confusion matrices indicate overall classifications that are very similar to those of HCs (see Fig. 2b, c, Tables S2, S3).

\section{Difference-BSMs}

Difference-BSMs are presented in Fig. 1d, e, f. The percentage of pixels painted by MDDnm compared to HC is presented in Table 2 . MDDnm painted only $63 \%$ as many pixels as those of $\mathrm{HC}$ overall, i.e., colored around half as many pixels as HCs. Applying the emotion classification of HC LDA on MDDnm reached an accuracy of $28 \%$ and of $26 \%$ in MDDm - both accuracy rates were still above chance level.

\section{Discussion}

The aim of this study was to analyze bodily sensations in emotion experience in healthy and depressed participants. On two body silhouettes presented during an emotion induction, participants colored the regions where they felt an increase or decrease in activation. We were able to conceptually replicate Nummenmaa and colleagues' (2014) results in healthy participants using a different stimulus set. Qualitatively observed, disgust seemed to be associated with activation in stomach, oesophagus and throat, while happiness was associated with strong activation in heart region and face. Sad pictures evoked stronger activation in heart, stomach and head region and fear a diffuse activation in legs, arms, chest and head. Compared to Nummenmaa and colleagues' (2014) results for emotion induction through movies, differences between results are only observable for sadness. In contrast to our results, their participants showed no activation in the stomach region and a deactivation in the legs. HC showed significantly distinct activation patterns for happiness, disgust and a neutral emotional state. Classification for fear and sadness was above chance level, though sadness was more often classified as happiness while fear was more often classified as neutral. Strikingly, especially compared to the original study by Nummenmaa and colleagues (2014), almost no deactivation reached significance. Nonetheless, Nummenmaa and colleagues found a similar reaction pattern when using emotional movies as an induction method. The misclassification of sadness as happiness might be caused by high activation in the chest and face regions in both states resulting from the pictures chosen, as both picture groups contained only pictures with social situations. The higher misclassification of fear as a neutral emotional state might be caused by a failed induction of fear through mildly arousing pictures. As such, a striking problem associated with emotion induction with pictures is that some emotions, such as fear and anger, are not easily inducible (Mikels et al. 2005). One might speculate, for example, that feelings of anger involve an offense against the self, which is not elicitable with non-self-related pictures (Lazarus 1991). Nummenmaa and colleagues, who had a similar problem when using movies, refrained from using anger stimuli. In the current study, anger pictures were used, but we were not able to induce distinct bodily sensation patterns for anger.

In addition to the conceptual replication of Nummenmaa and colleagues' results, the emBODY tool was also conducted with a depressed sample with or without current medication intake. Contrary to expectations, BSMs were found to be distinguishable among the depressed sample with a classification accuracy as high as in the HC group. In MDDnm, as in HCs, BSMs did not show deactivation responses that were significantly different from zero. However, activation was weaker than in the HC group, indicating either less activation during emotion processing in 
individuals with depression or reduced access to bodily sensations. Classifications were similar to $\mathrm{HC}$, with the exception that prediction of sadness was not above chance level and was more often misclassified as happiness. Sadness might therefore not be distinctly felt in depression. Nevertheless, as similar results were seen in the $\mathrm{HC}$ sample, no firm conclusion can be drawn.

In disgust, neutral emotional states and happiness, difference maps between $\mathrm{HC}$ and MDDnm were colored sparsely, whereas in sadness and fear, differences appeared to be present. In fear, less activation in the legs was present in the MDDnm compared to the HC sample. This result might be explained by the theory of learned helplessness (Maier and Seligman 2016); in depression, emotions that are typically associated with an impulse to escape might no longer be associated with its stimulating nature. With regard to sadness, the stomach and chest regions show less activation in MDDnm compared to HCs. It might be that blunted reactivity to stimuli underlies this finding.

It is noteworthy that the classification accuracy for MDDnm was only reduced by $6 \%$ after the LDA results of the HC sample were applied compared to the LDA results of the MDDnm itself. In line with this result, studies using IAPS pictures to compare healthy individuals with individuals with depression have failed to find differences in picture processing between college students with more or less depressive symptoms using self-report and autonomic arousal measures (Sloan and Sandt 2010). The weaker activation in depression compared to HCs is furthermore in line with findings from interoception studies, which have found that individuals with depression show deficits in IA (Eggart et al. 2019).

We conclude that no strong differences exist between HCs and MDDnm in the bodily sensations for all emotions, except for fear and sadness. Contrary to Torregrossa and colleagues' (2018) results in schizophrenia, depressed individuals show distinguishable activation patterns but less activation overall. Consequently, emotion experience seems to be differentially affected by the two mental disorders.

In addition, an exploratory aim of the current study was to examine the effect of medication on bodily sensations in depression. Again, classification accuracy was high, indicating that individuals with depression and medication intake felt distinct bodily sensations for different emotions. However, the MDDm sample was the only group that experienced strong feelings of deactivation while viewing the pictures. Difference maps showed less activation in all body parts except for the heart, stomach and head region compared to MDDnm and HCs. Classification accuracy was good for disgust, happiness, fear and neutral. Sadness was again more often misclassified as happiness.

Few studies have been conducted on the effect of antidepressant medication on emotion processing. Among those,
Read and colleagues (2014) reported that $60 \%$ of adults who took antidepressant medication reported feeling emotionally numb. Other studies have found similar results, including emotional blunting (e.g., Price et al. 2009) or difficulties identifying feelings (Kajanoja et al. 2018). These latter results are partially reinforced by the current findings. BSMs could be accurately separated for different emotions among MDDm, but the way they experienced their bodies during emotion induction differed strongly from HCs and MDDnm. Overall, the MDDm group sensed a decrease in bodily activation compared to the MDDnm and the HC samples. This might be associated with a feeling of numbness, as reported by Read and colleagues. Nevertheless, it cannot be ruled out that while the two depressed samples currently show equal symptom severity, the medicated group might have been more depressed before taking antidepressants. Therefore, the two groups might not be directly comparable even though they show equal symptom severity during the study. In order to draw firm conclusions, more research in this area is needed.

The current study has several limitations. In order to interpret effects found for depressed individuals taking medication and to analyze the impact of different types of antidepressant medication, a larger sample size is necessary. Several participants in the MDDm group took more than one antidepressant, which made it even more difficult to assess the exact influence of antidepressants. Therefore, results related to differences between MDDm and MDDnm should be considered as exploratory. Moreover, the selection of mildly intense pictures with a strong focus on social situations might have biased the results. Future studies that investigate body sensations in depression should utilize additional methods of inducing emotions and use larger stimulus sets and sample sizes determined with a thorough power analysis in order to present a conceptual replication that complies with highest standards for replication studies. Furthermore, in order to better understand BSMs it might be necessary to analyze physiological makers associated with them and introduce manipulation checks that clarify whether an emotion is felt at all. The impact of comorbid mental disorders and possibly confounding variables like amount of smoking or exercising remain important to consider as well. Of those comorbid mental disorders, in addition to anxiety, substance abuse should be kept in mind as substances alter body sensations as well (Verdejo-Garcia et al. 2012).

\section{Conclusion}

In the current study, distinct bodily activation patterns for different emotions induced by emotion-eliciting pictures were found in healthy subjects. Additionally, it was shown that individuals with depression, even though they showed 
distinct bodily activation patterns for different emotions, perceived less bodily sensations in emotional experiences, especially regarding sadness and fear. Weaker bodily sensations are in line with emotion research in depression that has found reduced negative and positive responsiveness in depression (Bylsma et al. 2008). The reduced bodily sensations indicate that it might be useful to support psychotherapeutic approaches that ameliorate body awareness in the cognitive behavioral therapy of depression. Approaches that already exist are for example mindfulness based cognitive therapy for depression (Segal et al. 2018), emotion focused therapy (Greenberg 2004) or exercising in depression (Mead et al. 2008). Moreover, we found that antidepressant medication seems to have a considerable impact on bodily sensations in emotion, but further research is needed to understand the nature of this effect in more detail. Overall, results indicate that increasing body awareness might be an important target in der treatment of patients with major depression.

Acknowledgements We thank Marie-Kristin Apel and Malica Rutzen for their support.

Author Contributions All authors contributed to the study conception and design. Material preparation and data collection were performed by AS and LA. Data analysis was performed by NL and BB. The first draft of the manuscript was written by NL and all authors commented on previous versions of the manuscript. All authors read and approved the final manuscript.

Funding Open Access funding enabled and organized by Projekt DEAL.

\section{Compliance with Ethical Standards}

Conflict of Interest The authors declare that they have no conflict of interest.

Ethical Approval Ethics approval was obtained from the ethics committee of Witten/Herdecke University.

Informed Consent Informed consent was obtained from all individual participants included in the study.

Animal Rights No animal studies were carried out by the authors for this article.

Open Access This article is licensed under a Creative Commons Attribution 4.0 International License, which permits use, sharing, adaptation, distribution and reproduction in any medium or format, as long as you give appropriate credit to the original author(s) and the source, provide a link to the Creative Commons licence, and indicate if changes were made. The images or other third party material in this article are included in the article's Creative Commons licence, unless indicated otherwise in a credit line to the material. If material is not included in the article's Creative Commons licence and your intended use is not permitted by statutory regulation or exceeds the permitted use, you will need to obtain permission directly from the copyright holder. To view a copy of this licence, visit http://creativecommons.org/licenses/by/4.0/.

\section{References}

Beck, A. T., Steer, R. A., \& Brown, G. K. (1996). Manual for the beck depression inventory-II. San Antonio, TX: The Psychological Corporation. https://doi.org/10.1007/978-1-4419-1005-9_441.

Berking, M., \& Wupperman, P. (2012). Emotion regulation and mental health: Recent findings, current challenges, and future directions. Current Opinion in Psychiatry, 25(2), 128-134. https://doi. org/10.1097/YCO.0b013e3283503669.

Bylsma, L. M., Morris, B. H., \& Rottenberg, J. (2008). A meta-analysis of emotional reactivity in major depressive disorder. Clinical Psychology Review, 28(4), 676-691. https://doi.org/10.1016/j. cpr.2007.10.001.

Cannon, W. B. (1927). The James-Lange theory of emotions: A critical examination and an alternative theory. American Journal of Psychology, 39, 106-124. https://doi.org/10.2307/1415404.

Craig, A. D. (2002). How do you feel? Interoception: The sense of the physiological condition of the body. Nature Reviews Neuroscience, 3(8), 655-666. https://doi.org/10.1038/nrn894.

Craig, A. D. (2003). Interoception: The sense of the physiological condition of the body. Current Opinion in Neurobiology, 13(4), 500-505. https://doi.org/10.1016/S0959-4388(03)00090-4.

Damasio, A., \& Carvalho, G. B. (2013). The nature of feelings: Evolutionary and neurobiological origins. Nature Reviews Neuroscience, 14(2), 143-152. https://doi.org/10.1038/nrn3403.

Eggart, M., Lange, A., Binser, M. J., Queri, S., \& Müller-Oerlinghausen, B. (2019). Major depressive disorder is associated with impaired interoceptive accuracy: A systematic review. Brain sciences, 9(6), 131. https://doi.org/10.3390/brainsci9060131.

Farb, N., Daubenmier, J., Price, C. J., Gard, T., Kerr, C., Dunn, B. D., \& Mehling, W. E. (2015). Interoception, contemplative practice, and health. Frontiers in Psychology, 6, 763. https://doi. org/10.3389/fpsyg.2015.00763.

Furman, D. J., Waugh, C. E., Bhattacharjee, K., Thompson, R. J., \& Gotlib, I. H. (2013). Interoceptive awareness, positive affect, and decision making in major depressive disorder. Journal of Affective Disorders, 151(2), 780-785. https://doi.org/10.1016/j. jad.2013.06.044.

Gendron, M., \& Feldman Barrett, L. (2009). Reconstructing the past: A century of ideas about emotion in psychology. Emotion review, 1(4), 316-339. https://doi.org/10.1177/1754073909338877.

Ginzburg, K., Tsur, N., Barak-Nahum, A., \& Defrin, R. (2013). Body awareness: Differentiating between sensitivity to and monitoring of bodily signals. Journal of Behavioral Medicine, 37, 564-575. https://doi.org/10.1007/s10865-013-9514-9.

Greenberg, L. S. (2004). Emotion-focused therapy. Clinical Psychology and Psychotherapy, 11(1), 3-16. https://doi.org/10.1002/ cpp.388.

Hahs-Vaughn, D. L. (2017). Applied multivariate statistical concepts. New York, USA: Routledge.

James, W. (1884). What is emotion. Mind, 9, 188-205. https://www. jstor.org/stable/2246769.

Kajanoja, J., Scheinin, N. M., Karukivi, M., Karlsson, L., \& Karlsson, H. (2018). Is antidepressant use associated with difficulty identifying feelings? A brief report. Experimental and Clinical Psychopharmacology, 26(1), 2-5. https://doi.org/10.1037/pha0000165.

Kreibig, S. D. (2010). Autonomic nervous system activity in emotion: A review. Biological Psychology, 84, 394-421. https://doi. org/10.1016/j.biopsycho.2010.03.010.

Kühner, C., Bürger, C., Keller, F., \& Hautzinger, M. (2007). Reliabilität und validität des revidierten beck-depressionsinventars (BDI-II). Befunde aus deutschsprachigen stichproben [Reliability and validity of the revised Beck-Depression inventory (BDI-II). Findings from a German-speaking sample]. Der Nervenarzt, 78, 651-656. https://doi.org/10.1007/s00115-006-2098-7. 
Lang, P. J., Bradley, M. M., \& Cuthbert, B. N. (2008). International affective picture system (IAPS): Affective ratings of pictures and instruction manual. Technical report A-8. Gainesville, FL: University of Florida.

Lazarus, R. (1991). Emotion and adaptation. New York: Oxford University Press.

Maier, S. F., \& Seligman, M. E. (2016). Learned helplessness at fifty: Insights from neuroscience. Psychological review, 123(4), 349367. https://doi.org/10.1037/rev0000033.

Mauss, I. B., Levenson, R. W., McCarter, L., Wilhelm, F. H., \& Gross, J. J. (2005). The tie that binds? Coherence among emotion experience, behavior, and physiology. Emotion, 5(2), 175-190.

Mauss, I. B., \& Robinson, M. D. (2009). Measures of emotion: A review. Cognition and Emotion, 23(2), 209-237. https://doi. org/10.1080/02699930802204677.

Mead, G. E., Morley, W., Campbell, P., Greig, C. A., McMurdo, M., \& Lawlor, D. A. (2008). Exercise for depression. Cochrane Database of Systematic Reviews. https://doi.org/10.1002/14651858. CD004366.

Mikels, J. A., Fredrickson, B. L., Larkin, G. R., Lindberg, C. M., Maglio, S. J., \& Reuter-Lorenz, P. A. (2005). Emotional category data on images from the international affective picture system. Behavior Research Methods, 37(4), 626-630.

Niedenthal, P. M. (2007). Embodying emotion. Science, 316(5827), 1002-1005. https://doi.org/10.1126/science.1136930.

Nummenmaa, L., Glerean, E., Hari, R., \& Hietanen, J. K. (2014). Bodily maps of emotions. PNAS, 111(2), 646-651. https://doi. org/10.1073/pnas.1321664111.

Paulus, M. P., \& Stein, M. B. (2010). Interoception in anxiety and depression. Brain Structure and Function, 214, 451-463. https:// doi.org/10.1007/s00429-010-0258-9.

Price, J., Cole, V., \& Goodwin, G. (2009). Emotional side-effects of selective serotonin reuptake inhibitors: Qualitative study. British Journal of Psychiatry, 195, 211-217. https://doi.org/10.1192/bjp. bp.108.051110.

Read, J., Cartwright, C., \& Gibson, G. (2014). Adverse emotional and interpersonal effects reported by 1829 new zealanders while taking antidepressants. Psychiatry Research, 216(1), 67-73. https ://doi.org/10.1016/j.psychres.2014.01.042.

Saarimäki, H., Ejtehadian, L. F., Glerean, E., Jääskeläinen, I. P., Vuilleumier, P., Sams, M., \& Nummenmaa, L. (2018). Distributed affective space represents multiple emotion categories across the human brain. Social Cognitive and Affective Neuroscience, 13(5), 471-482. https://doi.org/10.1093/scan/nsy018.

Schandry, R. (1981). Heart beat perception and emotional experience. Psychophysiology, 18(4), 483-488.

Segal, Z. V., Williams, J. M. G., \& Teasdale, J. D. (2018). Mindfulnessbased cognitive therapy for depression. New York, NY: Guilford Publications.

Simmons, J. P., Nelson, L. D., \& Simonsohn, U. (2011). False-positive psychology: Undisclosed flexibility in data collection and analysis allows presenting anything as significant. Psychological Science, 22(11), 1359-1366. https://doi.org/10.1177/0956797611417632.

Sloan, D. M., \& Sandt, A. R. (2010). Depressed mood and emotional responding. Biological Psychology, 84(2), 368-374. https://doi. org/10.1016/j.biopsycho.2010.04.004.

Torregrossa, L. J., Snodgress, M. A., Hong, S. J., Nichols, H. S., Glerean, E., Nummenmaa, L., \& Park, S. (2018). Anomalous bodily maps of emotions in schizophrenia. Schizophrenia bulletin. https://doi.org/10.1093/schbul/sby179.

Verdejo-Garcia, A., Clark, L., \& Dunn, B. D. (2012). The role of interoception in addiction: A critical review. Neuroscience and Biobehavioral Reviews, 36(8), 1857-1869.

Wittchen, H.-U., Wunderlich, U., Gruschwitz, S., \& Zaudig, M. (1997). Strukturiertes klinisches interview für DSM-IV [Structured clinical interview for DSM-IV]. Göttingen: Hogrefe. https://doi. org/10.1026//0084-5345.28.1.68.

Publisher's Note Springer Nature remains neutral with regard to jurisdictional claims in published maps and institutional affiliations. 